Stefanie Rhein

Lebensstil und Umgehen mit Umwelt 
SOZIALWISSENSCHAFT 
Stefanie Rhein

\section{Lebensstil und Umgehen mit Umwelt}

Empirisch-kultursoziologische Untersuchung zur Ästhetisierung des Alltags

Mit einem Geleitwort von Prof. Dr. Werner Heinrichs

Deutscher Universitäts-Verlag 
Bibliografische Information Der Deutschen Bibliothek

Die Deutsche Bibliothek verzeichnet diese Publikation in der Deutschen Nationalbibliografie; detaillierte bibliografische Daten sind im Internet über <http://dnb.ddb.de> abrufbar.

Dissertation Pädagogische Hochschule Ludwigsburg, 2005

\section{Auflage Januar 2006}

Alle Rechte vorbehalten

(C) Deutscher Universitäts-Verlag/GWV Fachverlage GmbH, Wiesbaden 2006

Lektorat: Ute Wrasmann / Britta Göhrisch-Radmacher

Der Deutsche Universitäts-Verlag ist ein Unternehmen von Springer Science+Business Media. www.duv.de

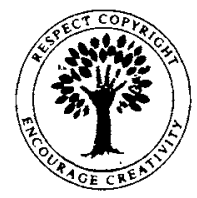

Das Werk einschließlich aller seiner Teile ist urheberrechtlich geschützt. Jede Verwertung außerhalb der engen Grenzen des Urheberrechtsgesetzes ist ohne Zustimmung des Verlags unzulässig und strafbar. Das gilt insbesondere für Vervielfältigungen, Übersetzungen, Mikroverfilmungen und die Einspeicherung und Verarbeitung in elektronischen Systemen.

Die Wiedergabe von Gebrauchsnamen, Handelsnamen, Warenbezeichnungen usw. in diesem Werk berechtigt auch ohne besondere Kennzeichnung nicht zu der Annahme, dass solche Namen im Sinne der Warenzeichen- und Markenschutz-Gesetzgebung als frei zu betrachten wären und daher von jedermann benutzt werden dürtten.

Umschlaggestaltung: Regine Zimmer, Dipl.-Designerin, Frankfurt/Main

Druck und Buchbinder: Rosch-Buch, ScheBlitz

Gedruckt auf säurefreiem und chlorfrei gebleichtem Papier

Printed in Germany

ISBN 3-8350-6016-3 


\section{Geleitwort}

Der vom persönlichen Geschmack geprägte Lebensstil scheint in immer mehr Bereichen des Alltags zum zentralen Entscheidungs- und Unterscheidungskriterium geworden zu sein: Man wählt für sich das aus, was gefällt und zum eigenen Stil passt - egal, ob es sich dabei um Musik-CDs, die Wahl des Verkehrsmittels, den Theaterbesuch oder die Inneneinrichtung der Wohnung handelt. Auch vorderhand nicht „ästhetische“ Alltagssituationen scheinen zunehmend ,ästhetisiert" zu werden und das meint, sie werden nicht nach ihrem praktischen Nutzen, sondern nach ihrer Erlebnisqualität und ihrer Eignung für einen auf Differenzierung ausgerichteten Lebensstil bewertet.

Entsprechend boomen kultursoziologische Lebensstilkonzepte nicht nur in den Kulturwissenschaften, sondern auch in zumindest scheinbar kulturfernen Bereichen wie beispielsweise dem Ökomarketing oder der Umweltpolitik. Jedoch scheint gerade in diesen eher kulturfernen Anwendungsbereichen oftmals eine theoretische und empirische Reflexion des Konzepts in Bezug auf genau diesen Bereich zu fehlen. Die Frage, wie weitreichend und umfassend die Ästhetisienung des Alltags tatsächlich ist, wird kaum gestellt.

Stefanie Rhein setzt in ihrer interdisziplinär angelegten Dissertation, die im Schnittpunkt von Kultur- und Umweltsoziologie, Kulturmanagement und Umweltbildung angesiedelt ist, genau an dieser Stelle an: Ausgehend von einer breiten theoretischen Basis untersucht sie empirisch, ob auch ein nicht vorderhand ästhetischer Alltagsbereich wie der des Umgehens mit Umwelt (z. B. Umwelteinstellung, Umweltwissen, Umwelthandeln) nach Kriterien der Erlebnisqualität und der Lebensstildifferenzierung gestaltet wird und damit unter das (vermeintliche) Verdikt der Ästhetisierung des Alltags fällt - oder ob hier möglicherweise ganz andere Handlungs- und Bewertungsschemata eine Rolle spielen. Anders formuliert lautet die Ausgangsfrage: Lassen sich Umwelt im ökologischen Verständnis sowie Umweltschutz und Umwelterziehung als Glücks- oder Spaßmoment der Erlebnisgesellschaft positionieren oder entziehen sie sich diesem modischen Denkmuster?

Die Untersuchung ist damit zum einen für die ökologisch orientierten Forschungs- und Anwendungsdisziplinen relevant, weil aus ihr Erkenntnisse abgeleitet werden können, die beispielsweise in der Umwelterziehung von Relevanz sein dürften. Zum anderen ist die Studie aber vor allem von kulturwissenschaftlicher Brisanz: Wenn sich das Umgehen mit Umwelt dem Schema der Ästhetisierung entzieht, dann stellt dies das kultursoziologische Paradigma von der generellen Ästhetisierung des Alltags in einer individualisierten Gesellschaft in Frage. Viele der Bewertungen und Analysen, die in jüngster Zeit pauschal unter dem Gesichtspunkt des Lebensstilkonzepts und dem Verdikt der Ästhetisierung erfolgten, müssten dann möglicherweise künftig differenzierter gesehen werden. Das hätte beispielsweise nachhaltige Folgen für viele aktuelle Marketingkonzepte, die ausschließlich die Ästhetisierungsthese, die These von der Erlebnisqualität und das Lebensstilmodell als Ausgangsbasis nehmen. Vor allem im Kulturmarketing, wo die Erlebnisorientienung und das Lebensstilmodell zur Zeit als Konzeptionsbasis unangefochten dominieren, könnte dies zu einer größeren Offenheit für andere Konzepte und damit auch wieder zu differenzierteren Marketingstrategien führen. Ange- 
sichts der in den letzten Jahren zu beobachtenden, zunehmenden Verengung der wissenschaftlichen Analyse auf nur ein Modell, darf man die mit der Untersuchung von Stefanie Rhein sich abzeichnende Öffnung als einen großen Gewinn bewerten.

Prof. Dr. Werner Heinrichs 


\section{Dank}

Bei der Arbeit an meiner Dissertation wurde ich von vielen Seiten auf unterschiedlichste Weise unterstützt:

An vorderster Stelle möchte ich mich bei Frau Prof. Dr. Renate Müller und Herrn Prof. Dr. Werner Heinrichs bedanken, die meine Arbeit mit großem Engagement betreut haben.

$\mathrm{Zu}$ danken habe ich auch den Institutionen, die mich finanziell und durch die Bereitstellung notwendiger Infrastruktur unterstützt haben: Deutsche Forschungsgemeinschaft Bonn (DFG), Landesgraduiertenförderung Baden-Württemberg und Pädagogische Hochschule Ludwigsburg. Mein Dank geht an Frau PD Dr. Gisela Böhm als Leiterin des DFG-Projekts „Umweltrisiken: Affekt, Bewertung und Verhalten", in dessen Kontext diese Arbeit entstand, und an das Projektteam Sonja Schlegel, Petra Baisch, Anja Vocilka und Susanne Jaggy. Außerdem danke ich den Befragungsteilnehmern und -teilnehmerinnen sowie den Institutionen und Personen, die uns bei der Realisierung der Befragungen unterstützten.

Für das Korrekturlesen des Manuskripts, für hilfreiche inhaltliche Diskussionen und Anmerkungen geht mein Dank an Helmut Rhein, Patrick Glogner, Sonja Schlegel, Marc Calmbach und Achim Pietzcker. Außerdem danke ich meinen Eltern, meinen Geschwistern Christof und Matthias mit Iris Rhein, meinen Verwandten Rudolf und Sigrid Rhein und meinen Freunden insbesondere Ahlie Schaubel, Steph Schneider, Thomas Sattig, Jan-Torsten Kohrs, Thomas Koch, Philippe Frowein, Familie Haller/ Dobelmann und allen aus dem Arbeitsraum Tübinger Straße-, die mir mit Rat und Tat zur Seite standen.

Stefanie Rhein 


\section{Inhaltsverzeichnis}

0

1 Stand der Forschung: Kultursoziologische und ökologische Lebensstilforschung

1.1 Zur Wahl und Verwendung der Begriffe: Umgehen mit Umwelt und Umweltbezug

1.1.1 Zur Bevorzugung des Begriffs „Umgehen mit Umwelt“ gegenüber dem Begriff „Umweltbewusstsein“ in dieser Arbeit

1.1 .2 Umweltbezug

1.2 Stand der Forschung: Umgehen mit Umwelt und (kultur-)soziologische Lebensstilforschung

1.3 Ökologische Lebensstilforschung

1.3.1 Umweltbezogene Lebensstile der letzten Jahrzehnte 13

1.3.2 Von der Umweltbewusstseinsforschung zur ökologischen 15

1.3.3 Stand der Forschung: Erkenntnisse und Stränge der ökologischen $\quad 18$

$1.4 \quad$ Verortung der vorliegenden Studie 24

2 Das Umgehen mit Umwelt als Teil des Lebensstils 29

$2.1 \quad$ Grundannahmen des Symbolischen Interaktionismus 29

2.2 Lebensstiltheorie und die Zentralität von Kultur und Ästhetik 30

2.2.1 Die Kriterien der Identifizierbarkeit und der Überhöhung 32

2.2.2 Lebensstile als Ausdruck sozialer und ästhetischer Differenzierungen 33

2.2.3 Soziale Verortung über ästhetische Präferenzen: Vom Wandel 34

2.3 Die Dimensionen des hier verwendeten Lebensstilmodells 37

2.4 Wahlfreiheit revisited: Zum Verhältnis von Lebensstilen und 39

$\begin{array}{lll}2.4 .1 & \text { Wahlfreiheit } & 40\end{array}$

2.4.1.1 Wahlfreiheit als eine Frage der Ressourcen 40

2.4.1.2 Das Phänomen der Ungleichzeitigkeit 40

2.4.1.3 Zwischen bewusster Stilisierung und Selbstbegrenzung 42

2.4.2 Lebensstile und soziodemographische Merkmale 43

2.4.3 Soziodemographische Merkmale und das Umgehen mit Umwelt 44

$3 \quad$ Sozialisation, Identität und Lebensstil $\quad 47$

3.1 Träger von Lebensstilen: Individuen oder soziale Aggregate?

3.2 Die „Scharnierfunktion“ des Lebensstils 48

3.2.1 Das Identitätskonzept des Symbolischen Interaktionismus 48 
3.2.2 Lebensstile: Individualität und Gemeinsamkeit 50

$\begin{array}{lll}\text { 3.2.3 Sozialisation als Selbstsozialisation } & 51\end{array}$

3.2.3.1 Das Konzept der Selbstsozialisation 51

3.2.3.2 Überlegungen zur umweltbezogenen Sozialisation im Kontext 53 des Selbstsozialisationsansatzes

4 Die sinn- und wertebezogene Dimension der Lebensstile 57

4.1 Werte, Wertewandel, ökologischer Diskurs 60

4.1.1 Wertewandel: Linearer Wandel oder Pluralisienung? 61

4.1.2 Der ökologische Diskurs als sozialer Bezugspunkt 62

4.2 Bedeutungszuschreibung und Zeichenstrukturierung als 64

$\begin{array}{lll}4.3 & \text { Deutungsgemeinschaften } & 66\end{array}$

5 Zusammenfassung: Theorie und Hypothesen 69

5.1 Kulturell-ästhetische Orientierungen, Wertvorstellungen und 69

$5.2 \quad$ Umgehen mit Umwelt 71

$\begin{array}{lll}5.3 & \text { Die soziale Dimension des Umgehens mit Umwelt } & 72\end{array}$

5.4 Soziodemographische Merkmale, die kulturell-ästhetische und die $\quad 74$

$\begin{array}{lll}5.5 & \text { Soziokulturelle Kontexte als Deutungskontexte } & 77\end{array}$

6 Untersuchungsdesign, Methode und Operationalisierungen 79

$\begin{array}{lll}6.1 & \text { Besonderheiten des Designs } & 79\end{array}$

6.2 Die Auswahl der Erhebungsmethode 80

6.3 Strukturierungsgrad des Fragebogens 81

6.4 Fragebogensegmente zur Operationalisierung der Hypothesen 82

6.4.1 Kultureller Geschmack und kulturelle Umgehensweisen 82

$\begin{array}{lll}6.4 .2 & \text { Soziodemographische Merkmale } & 83\end{array}$

$\begin{array}{lll}\text { 6.4.3 Wertvorstellungen: Wichtige Lebensaspekte } & 84\end{array}$

$\begin{array}{lll}6.4 .4 & \text { Umgehen mit Umwelt } & 87\end{array}$

$\begin{array}{lll}\text { 6.4.4.1 Umweltwissen und Umwelteinstellung } & 87\end{array}$

6.4.4.2 (Institutionelle) Beschäftigung mit dem Thema Umwelt/ Natur 89

6.4.4.3 Umweltverhalten $\quad 89$

6.4.4.4 Umweltbezug: Allgemeine Ausrichtungen des Umgehens mit Umwelt 91

6.4.4.5 Die soziale Dimension des Umgehens mit Umwelt 92

6.5 Das Problem der sozialen Erwünschtheit im Zusammenhang mit einer 93 Befragung zum Thema „Umgehen mit Umwelt“

6.6 Aufbau des Fragebogens 96

$\begin{array}{lll}6.7 & \text { Probandenauswahl und -gewinnung } & 97\end{array}$ 

und Befragungsbedingungen

$8.1 \quad$ Kulturell-ästhetische Orientierungen und kulturelle Aktivitätenarten 103

$\begin{array}{lll}\text { 8.2 Kulturell-ästhetische Orientierungen und ihre werte- und sinnbezogene } & 108\end{array}$ Dimension

$\begin{array}{lll}\text { 8.2.1 Struktur der Wertvorstellungen } & 108\end{array}$

8.2.2 Exkurs: Der 11. September 2001 im Kontext dieser Arbeit 111

8.2.3 Kulturell-ästhetische Orientierungen und Wertvorstellungen 112

8.3 Kulturell-ästhetische Orientierungen und soziodemographische Merkmale 115

8.3.1 Soziodemographisch bedingte kulturell-ästhetische Affinitäten 115

8.3.2 Soziodemographische Merkmale und kulturell-ästhetische 119 Orientierungen: Bewertung der Befunde vor dem Hintergrund anderer kultursoziologischer Studien

8.4 Zusammenfassung: Kulturell-ästhetische Orientierungen, Wertvorstellungen und soziodemographische Merkmale

$9 \quad$ Kulturelle Typen

$\begin{array}{lll}9.1 & \text { Kulturelle Typen und ihre Wertvorstellungen } & 124\end{array}$

9.2 Kulturelle Typen und soziodemographische Merkmale 125

9.3 Zusammenfassung: Kulturelle Typen, ihre Wertvorstellungen und 126

$10 \quad$ Umgehen mit Umwelt 127

10.1 Umweltorientierungen: Ausrichtungen des Umgehens mit Umwelt 127

10.2 Einzelne Aspekte des Umgehens mit Umwelt 130

10.2.1 Umwelteinstellung und Umweltwissen 130

10.2.2 Die Struktur des Umweltverhaltens 132

$\begin{array}{lll}\text { 10.2.3 Beschäftigung mit dem Thema Umwelt/ Natur } & 137\end{array}$

10.3 Die Umweltorientierungen und die verschiedenen Aspekte des Umgehens 138 mit Umwelt

10.4 Von den Umweltorientierungen zu den Umwelttypen

11 Die sinn- und wertebezogene Dimension des Umgehens mit Umwelt 145

11.1 Wertvorstellungen und das Umgehen mit Umwelt 145

11.1.1 Wertvorstellungen und Umweltorientierungen 145

11.1.2 Wertvorstellungen und einzelne Aspekte des Umgehens mit Umwelt 148

11.2 Zusammenfassende Darstellung: Umgehen mit Umwelt und 152 Wertvorstellungen 
12.1 Die Facetten der sozialen Relevanz 155

12.2 Die soziale Relevanz der verschiedenen Umweltorientierungen $\quad 158$

12.2.1 Die soziale Relevanz von Umweltinvolvement, Umweltabwertung $\quad 158$

12.2.2 Umwelttypen und die soziale Relevanz ihres Umgehens mit Umwelt $\quad 160$

12.3 Zwischenstand: Die soziale Dimension des Umgehens mit Umwelt 161

$12.4 \quad$ Umgehen mit Umwelt im sozialen Kontext von Freundeskreis $\quad 162$

12.4.1 Gemeinschaft und Gemeinsamkeit: Umweltschutz in Freundeskreis $\quad 162$

12.4.2 Umwelttypen: Die soziale Relevanz ihrer Umweltorientierung und das 165 jeweilige Umgehen mit Umwelt in Freundeskreis und Familie

12.4.2.1 Die Umwelttypen und das Umgehen mit Umwelt in ihrem sozialen 165 Umfeld

12.4.2.2 Das soziale Umfeld der Umwelttypen und sein Einfluss auf die soziale $\quad 166$ Relevanz des Umgehens mit Umwelt

12.5 Die soziale Dimension der Umweltorientierungen und die konkrete Gestaltung des Umgehens mit Umwelt

12.6 Zusammenfassung: Die Frage nach der Stilhaftigkeit der Umweltorientierungen

13 Umgehen mit Umwelt: soziodemographische Merkmale, kulturell-ästhetische und wertebezogene Dimension

13.1 Soziodemographische Merkmale und das Umgehen mit Umwelt 175

13.2 Die kulturell-ästhetische Dimension des Umgehens mit Umwelt 178

13.2.1 Die kulturell-ästhetische Dimension der Umweltorientierungen $\quad 178$

13.2.2 Die kulturell-ästhetische Dimension der einzelnen Aspekte des 180

13.3 Umgehen mit Umwelt - eine Frage kulturell-ästhetischer Präferenzen? $\quad 183$

14 Soziodemographische Gruppierungen: Die wertebezogene und die kulturell-ästhetische Dimension ihrer Úmweltorientierungen

14.1 Soziodemographische Gruppen und die wertebezogene Dimension ihrer Umweltorientierungen

14.2 Soziodemographische Gruppen und die kulturell-ästhetische Dimension ihrer Umweltorientierungen

14.3 Zusammenschau: Die kulturell-ästhetische und die wertebezogene Dimension der Umweltorientierungen unter Berücksichtigung der soziodemographischen Merkmale 
15.1 Kulturelle Typen und die Ausrichtung ihres Umgehens mit Umwelt

15.2 Kulturelle Typen und die wertebezogene Dimension ihrer Umweltorientierungen

16 Umgehen mit Umwelt im Kontext kulturell-ästhetisch definierter Lebensstile: Zusammenfassung und Diskussion der Befunde

Literaturverzeichnis 


\section{Tabellenverzeichnis}

Tab. 1

Aspekte des Umweltbewusstseins $\quad 7$

Tab. 2

Vergleich alternativer und nachhaltiger Lebensstile

Tab. 3

Umweltmentalitäten (Tabelle nach Kuckartz 2002, 119)

Tab. 4

Alltagsästhetische Schemata im Überblick (Schulze 1997, 163)

Tab. 5

Milieusegmentierung der erwachsenen Bevölkerung der BRD

(alte Bundesländer) am Ende der 1980er Jahre (Schulze 1997, 165)

Tab. 6

Wertetypen (Schwartz 1992; Boehnke 2004, 115)

Tab. 7

Untersuchungsdesign

Tab. 8

Wichtigkeit verschiedener Lebensaspekte

Tab. 9

Fragebogensegment Umweltwissen/ Umwelteinstellung

Tab. 10

Beispiel-Items für allgemeine Verhaltensfragen

Tab. 11

Items zur allgemeinen Ausrichtung des Umgehens mit Umwelt

Tab. 12

Soziale Relevanz des Umgehens mit Umwelt

Tab. 13

Verteilung Berufsgruppen

Tab. 14

Zusammenhänge zwischen den soziodemographischen Variablen

Tab. 15.1

Struktur der kulturell-ästhetischen Orientierungen

104

Tab. 15.2

Uneindeutig auf die fünf Faktoren ladende Items

Tab. 16

Kulturell-ästhetische Orientierungen

Tab. 17

Kulturell-ästhetische Orientierungen und kulturelle Aktivitätenarten

Tab. 18

Struktur der Wertvorstellungen

Tab. 19

Wertebereiche

Tab. 20

Wichtigkeit der einzelnen Lebensaspekte

Tab. 21

Kulturell-ästhetische Orientierungen und Wertvorstellungen

Tab. 22

Wertvorstellungen und kulturell-ästhetische Orientierungen;

Tab. 23 schematische Darstellung

Tab. 24 Beschreibung der kulturell-ästhetischen Orientienungen in

Tab. 25 soziodemographischer Hinsicht

Tab. 32 
Tab. 35 Umgehen mit der Ressource Wasser 136

$\begin{array}{lll}\text { Tab. } 36 & \text { Umweltverhalten } & 137\end{array}$

Tab. 37 Beschäftigung mit dem Thema Umwelt/ Natur 138

Tab. 38 Umweltorientierungen und die verschiedenen Aspekte des Umgehens 140 mit Umwelt

$\begin{array}{lll}\text { Tab. } 39 & \text { Umweltorientierungen und Wertvorstellungen } & 147\end{array}$

$\begin{array}{lll}\text { Tab. } 40 \quad \text { Umgehen mit Umwelt und Wertvorstellungen } & 149\end{array}$

Tab. 41 Umweltverhalten und Wertvorstellungen 150

Tab. 42 Umgehen mit Umwelt: Wertvorstellungen und soziodemographische 153

Tab. 43 Die Struktur der sozialen Relevanz des Umgehens mit Umwelt 155

Tab. 44 Soziale Relevanz im Kontext von Freundeskreis und Familie 156

Tab. 45 Soziale Relevanz des Umgehens mit Umwelt 157

Tab. 46 Soziale Relevanz und soziale Funktionen der Umweltorientierungen 159

Tab. 47 Umweltschutz im Freundeskreis und in der Familie 163

Tab. 48 Umweltorientierung und das Umgehen mit Umwelt in Freundeskreis 164

Tab. 49 Umweltschutz im Freundeskreis und in der Familie 165

Tab. 50 Umwelttypen: Einzelkämpfertum und das Umgehen mit Umwelt in 166

Tab. 51 Umwelttypen: Soziale Relevanz des Umgehens mit Umwelt und das 167

Tab. 52 Soziale Relevanz und Umgehen mit Umwelt in der Gruppe der $\quad 170$

Tab. 53 Die soziale Relevanz des Umgehens mit Umwelt und die einzelnen Bereiche des Umweltverhaltens und Beschäftigung mit dem Thema Umwelt/ Natur (nur: Umweltinvolvierte)

Tab. 54 Soziodemographische Merkmaie und das Umgehen mit Umwelt

Tab. 55 Kulturell-ästhetische Orientierungen und Umgehen mit Umwelt

Tab. 56 Umweltinvolvement und Wertvorstellungen: soziodemographisch

Tab. 57 Umweltegoismus und Wertvorstellungen: soziodemographisch bedingte Zuordnungen

Tab. 58 Umweltabwertung und Wertvorstellungen: soziodemographisch bedingte Zuordnungen

Tab. 59 Soziodemographische Gruppierungen und die kulturell-ästhetische Dimension ihrer Umweltorientierungen

Tab. 60.1 Zusammenschau der Befunde hinsichtlich der Wertvorstellungen, der kulturell-ästhetischen Orientierungen und dem Umgehen mit Umwelt in den soziodemographischen Gruppen (1) 
Tab. 60.2 Zusammenschau der Befunde hinsichtlich der Wertvorstellungen, der 200 kulturell-ästhetischen Orientierungen und dem Umgehen mit Umwelt in den soziodemographischen Gruppen (2)

Tab. 61 Kulturelle Typen und die wertebezogene Verortung des Umweltinvolvements

Tab. 62 Kulturelle Typen und die wertebezogene Verortung des

Umweltegoismus 


\section{Abbildungsverzeichnis}

Abb. 1 Die drei Dimensionen des Lebensstils 38

Abb. 2 Circumplex-Struktur menschlicher Werthaltungen 58

(Schwartz 1992, 45; vgl. auch: Boehnke 2004, 115)

Abb. $3 \quad$ Kulturelle Typen und ihre kulturell-ästhetischen Orientierungen $\quad 123$

Abb. $4 \quad$ Umweltorientierungen der Umwelttypen 142

Abb. 5 Umwelttypen und soziale Relevanz des Umgehens mit Umwelt; 161 $\mathrm{N}=146(142)$

Abb. 6 Umweltinvolvement bzw. Umweltegoismus und kulturell-ästhetische Orientierungen: potentielle wertebezogene „Bedeutungsklammern“

Abb. $7 \quad$ Kulturelle Typen und ihre Umweltorientierung 201

Abb. $8 \quad$ Alter, Kulturtyp und Umweltinvolvement 202

Abb. 9 Wohnort, Kulturtyp u. Umweltinvolvement 203 